\title{
A Study on Classification of Sustainable and Unsustainable Countries
}

\author{
Xinglong Ren and Hong Fang \\ College of Science, Huazhong Agricultural University, Wuhan,China \\ *Corresponding author
}

\begin{abstract}
We construct an evaluation system of sustainable development countries using the method of Bayes, k-means. Based on the classification, the developing model can be categorized into self-sufficient, technology-dependent and funddependent countries. We also explores the sustainable development of Equatorial Guinea. Finally, we evaluate the effect of sustainability plan and highly effective strategies.
\end{abstract}

Keywords-coordination-sustainability model;

Bayes discriminan; $k$-means

\section{INTRODUCTION}

Balancing human needs with the earth's health has been a topic of considerable debate since the last half of the twentieth century. To reconcile the balance, the concept of sustainable development was defined in 1987 Brundtland Report. Lots of works have been done in sustainable development. It is our task to study sustainable development now.

We are required to develop a model which can distinguish whether a country is sustainable or not. It has to define when and how a country is sustainable. It can also show us the countries which are in bad need of support and intervention. After that, create a 20-year sustainable development plan which consists of programs, policies. Evaluate the effect 20year sustainability plan has on the country's sustainability measure created. Predict the change that will occur over the 20 years in the future by implementing the 20-year plan. The ultimate goal is to identify highly effective strategies to create a more sustainable world.

According to recent research, people are thinking highly of qualitative analysis in- stead of focusing on quantification analysis of sustainable development. AHP [20], fuzzy comprehensive evaluation, factor analysis, principle component analysis[17], neural net- work, DEA are common algorithms to evaluate sustainable development. However, the selection of weights in AHP is subjective. Factor analysis, principle component analysis is widely used to process highdimension data. The accuracy of fuzzy comprehensive evaluation is low due to fuzzy initial data. We will use discriminant analysis based on the above methods.

\section{MODELS}

\section{Sustainability of Different countries}

1) The indicator system of sustainable development countries: We choose three indicators: environment, economy and society to signify sustainable development [2]. To detail these indicators we choose carbon dioxide emission, forest coverage rate, marine protected area ( $\%$ of territorial waters), Life Expectancy Index, Education, Per Capita GNI and Per Capita GDP (Net ODA Received will not be use until next step). These factors illustrate the situation of local environmental quality, energy, livelihoods, human health respectively as the problem required .[4]

The Indicator System is divided into three layers called the bottom layer, the medium layer and the top layer.

The bottom layer is composed of seven elements: carbon dioxide emission (To sim- plify calculation, we abbreviate its initial value as $\mathrm{CO} 2$, similarly hereinafter), forest cov- erage rate (FCR), marine protected area ( $\%$ of territorial waters) (MPR), Life Expectancy Index (LE), Education (EI), Per Capita GNI (GNIPC) and Per Capita GDP (GDPPC) (Net ODA Received will not be use until next step). They have to be measured by correlative indexes. The algorithms of correlative indexes are as below. The results of these elements are calculated without dimension in that they range from 0 to 1 .

$$
\begin{aligned}
& C O_{2} I=\frac{C O_{2 \max }-C O_{2}}{C O_{2 \max }-C O_{2 \min }} \\
& F C R I=\frac{F C R-0}{100-0} \\
& M P R I=\frac{M P R-0}{100-0} \\
& L E I=\frac{L E-20}{83.2-20}
\end{aligned}
$$

$$
E I=\sqrt{\frac{M Y S I \cdot E Y S I}{0.951}}
$$




$$
G N I I_{P C}=\frac{\ln G N I_{P C}-\ln 100}{\ln 107711-\ln 100}
$$

$$
G D P I_{p c}=\frac{\ln G D P_{p c}-\ln G D P_{p c \min }}{\ln G D P_{p c \max }-\ln G D P_{p c \text { min }}}
$$

Note: MYSI=MYS/13.2 means the average educational years, $\mathrm{EYSI}=\mathrm{EYS} / 20.6$ means the expected educational years.

The medium layer consists of ecological environment (EE), economy (EC) and society (SC). The indicators are computed by the elements of the bottom layer. The computing equations are as bellow:

$$
E E I=\sqrt[3]{C O_{2} I \cdot F C R I \cdot M P R I}
$$

$$
S C I=\sqrt[3]{L E I \cdot E I \cdot I I}=H D I
$$

$$
E C I=G D P I_{p c}
$$

The indicators in the medium layer are geometric means of the indexes in the bottom layer. Using geometric means simulates the algorithm of Human Development Index (HDI) which is a useful indication of sustainable development [6].

The top layer is to judge the type of a given country by further calculation.

2) The classification of sustainable development: In this step, Bayes discriminant analysis is used to classify all the countries in the world to two groups: sustainable and unsustainable countries. We pick up some worldly accepted sustainable and unsustainable countries [7] as model countries. We grant eighty percent of the model countries as experiment set to provide discriminant evidence for Bayes method, the discriminant functions are as below:

$$
\begin{aligned}
& S_{1}=-29.15+66.22 \cdot S C I-3.80 \cdot E E I+10.26 \cdot E C I \\
& S_{2}=-10.76+39.19 \cdot S C I+11.75 \cdot E E I-13.26 \cdot E C I
\end{aligned}
$$

The rest twenty percent of the model countries are used to test the equations. The testing result shows that the equations fit the countries well.

Substitute data in above two equations, we obtain the value of (11) and (12). If the value of (11) is bigger than that of (12), then we can consider the country as a sustainable country. Otherwise, it is a unsustainable country.
The result demonstrates there are two main categories among the unsustainable countries. All the unsustainable countries may not be richer than the sustainable countries, while some of them can develop without economic aid for foreign countries, the essential help for them may be the technological and political assistance. We define this sort of countries as high-level-unsustainable countries. The rest countries are low-level-unsustainable countries. Technology can be a way for them to develop, but financial aid and intervention can help further.

The countries can also be classified into three developing types: self-sufficient, technology- dependent and funddependent countries. Self-sufficient countries can meet its need. Technology-dependent countries may develop better with technology assistance. Fund- dependent countries need fund-aid most.

3) When and how a county is sustainable or unsustainable: (13) is the result of the subtraction of (11) and (12). It can be viewed as a function that describes the sustainability of countries. When the calculation of (13) is greater than 0 , the country is in sustainable condition. Otherwise, it is in unsustainable condition. The coefficients reveal how much the indicators affect the sustainable development to some extent.

$$
\Delta \mathrm{S}=-18.39+27.03 \cdot \mathrm{SCI}-14.55 \cdot \mathrm{EEI}+23.52 \cdot \mathrm{ECI}(13)
$$

As we can see, the coefficients of society (SC) and economy (EC) influence sustainable development most. The larger these two values, the better the country develops. Ecological environment was thought to be the most important factor, however it plays inverse influence on sustainable development. The main reason was that the selection of indicators may not reveal all the factors universally. And another possible explanation is that there is possibility for some countries can improve sustain- ability by developing economy in cost of damaging environment.

\section{Coordination development model}

A 20-year plan has been made for Equatorial Guinea, a member of Least Developed Countries.

1) Searching for developing method: According to conclusion of Task 1, a country is sustainable when it is greater than 0 .Searching is processed based on, using society(SC), economy(EC) and ecological environment (EE), steps at 0.02 by MATLAB. The three indicators are dimensions which range from $0-1$. Extreme cases like (1-0-1) show up beyond expectation. This condition indicates people can develop economy and society and destroy environment at the same time which is unrealistic. To solve the problem, we introduce coordination development degree model (we abbreviate coordination development degree as CDD). The model is calculated as below:

$$
X_{i t}=\sum_{j=1}^{i} w_{i j} a_{i j t}
$$


Note: $a_{i j t} \in A$ is the dimension value of indicator $j$ for sub-system $i$ at time $t, x i t \in X$ is the development degree for sub-system $i$ at time $t$, wit is the weightgiventoindicator $j$ inthesub-system $i$.

$$
\bar{H}_{t}^{\prime}=\sqrt{\frac{\sum_{i=1}^{m}\left(x_{i t}-x_{i t}^{\prime}\right)^{2}}{\sum_{i=1}^{m} h_{i}^{2}}}\left(h_{i}=\max \left|x_{i t}-x_{i t}^{\prime}\right|\right)
$$

Note: $x_{i t}^{\prime} \in X^{\prime}$ is the ideal (maximum) value of development degree for sub-system $i . \bar{H}_{t}\left(X_{t}, X_{t}^{\prime}\right)(X)$ is the distance between real status and ideal coordination status at time $t$.

$$
\begin{aligned}
& c_{t}=\left(\sqrt{1-H_{t}^{\prime}}\right)^{k} \\
& x_{t}=\frac{1}{m} \sum_{i=1}^{m} x_{i t} \\
& d_{t}=\sqrt{x_{t} \cdot c_{t}}
\end{aligned}
$$

Note: $d_{t}$ is the systematic coordination development at time t.

In our calculation, the values of weights are scientific [13]. Three indicators from (13) are quoted from the results as 0.415 , $0.223,0.361$ of model 1 . Making k equals 1 , we obtain dt. Due to statistics analysis of our computation, we take 0.5 and 0.7 as critical values. If the value of a country is less than 0.5 , we take it as discordant country. Countries with value vary between 0.5 and 0.7 are medium-coordinated countries. Those with value greater than 0.7 are coordinated countries.

We integrate (13) in Task 1 and CCD in one model named coordination- sustainability model. We determine $\Delta S>0$ and $\mathrm{dt}>0.5$ as target. Sustainable countries may not reach coordinated status, so the value of $\mathrm{dt}$ should be 0.5 instead of 0.7.There are nearly 60,000 ideal developing method. This way of measuring sustainable countries complete the model in Task 1 .

2) Analysis of Equatorial Guinea: We choose Equatorial Guinea to study its developing model randomly in 48 Least Developed Countries. Its coefficients are 0.5586, 0.6953 and 0.1141. By calculating its Euclidean distance with all the ideal developing method, we choose the model with smallest distance as the most suitable developing model. The corresponding values are $0.7598,0.6006,0.2801$. We assume the increasing rate of each indicator will be same every year and Equatorial Guinea will accomplish its goal in all 20 years. And the country will turn into a sustainable country.

$$
T_{i}=C_{0 i}\left(1+x_{i}\right)^{k}
$$

Note $: T_{i}$ is the target value of indicator $i$ in twenty years. $C_{0 i}$ is the initial value of indicator $i . x_{i}$ is the average increasing rate of indicator $i$ in twenty years. $k$ equals 20 according to requirement.

\section{Evaluating THE EFFECT OF 20-YeAR SustainABILITY PLAN}

\section{A. Evaluation of the Plan}

In this process, we evaluate whether Equatorial Guinea can be sustainable or not in 20 years and predict how Equatorial Guinea changes. If the country reaches the increasing rate of society, environment and economy at $0.0155,-0.0073$ and 0.0457 strictly every year. will be greater than 0 in twenty years, which indicates Equatorial Guinea can be measured as a sustainable country twenty years from now on. The transition from low- level-unsustainable country to sustainable country is successful.

In fact, ideal situations hardly happen. Unexpected indexes affect the practical increasing rate. What's more, it is difficult to quantity these indicators. Therefore, we take these factors as a random influence factor. In order to simulate the effects of the random influence factor, we set variation range for the three indicators at $\pm 5 \%$ randomly, repeating 1,000 times. It turned out that Equatorial Guinea can be a sustainable country after 20 years.

With variation range getting larger, we study the range that can't ensure a sustainable country in twenty years. It means 50 simulations in 1,000 simulations can't ensure that $\Delta \mathrm{S}$ is greater than 0 . By several simulations, we obtain variation range at $\pm 12 \%$.Variation range of all the three indicators less than $\pm 12 \%$ can ensure the country is sustainable after twenty years.

\section{B. Evaluation of Highly Effective Strategies}

Without indicators selected by experts, the indicators for evaluation of highly effective strategies may be simple and subjective. To determine which programs or policies produce the greatest effect on the sustainability measure for the country, we measure it by society, environment, economy, coordination degree and ODA. Society, environment, economy, which are also the basic elements to evaluate sustainability. We also add coordination degree and ODA as indicators.

The algorithm of society, environment and economy can be seen in Task 1. The arithmetic of coordination degree can be acquired from Task 2.The algorithm of ODA can imitate equation (1). We use Radar chart to analyze and compare different policies.

\section{Merit and Demerit}

Define abbreviations and acronyms the first time they are used in the text, even after they have been defined in the abstract. Abbreviations such as IEEE, SI, MKS, CGS, sc, dc, and rms do not have to be defined. Do not use abbreviations in the title or heads unless they are unavoidable. 


\section{Conclusions}

Our solution involves a series of models. The Indicator System of Sustainable Development has several indicators divided into three layers. They are calculated step by step and we get three indicators: ecological environment, economy and society. The three indicators are used in Bayes discriminant analysis to determine the weights in discriminant functions. Countries are divided into sustainable and unsustainable countries by Bayes discriminant function. We discover there is a gap in unsustainable countries, so the above mentioned three indicators and ODA are used in K-means algorithm. In this step, unsustainable countries are classified into high-levelunsustainable countries and low- level-unsustainable countries For any country, if its value of discriminant function S1 is larger than that of discriminant function S2, we consider the country as a sustainable country.

We search all possible methods for various countries to make their own decisions. We integrate $\Delta \mathrm{S}>0$ and threshold of coordination development degree at 0.5 as coordination developing model to determine coordinated and sustainable developing method. To study specific developing procedure, we choose an un- sustainable Least Developed Country Equatorial Guinea. According to our evaluation system of sustainable countries, Equatorial Guinea is a low-levelunsustainable country. Such resources as oil are the main income of Equatorial Guinea. By calculating Euclidean distance with all the developing models, we choose the model with smallest distance as the most suitable method. In our model, Equatorial Guinea can grow into a sustainable one. It will be a sustainable country in twenty years at increasing rates of society, environment and economy as $0.016,0.0073$ and 0.046. As we discussed before, Equatorial Guinea change the target year of becoming sustainable, the increasing rate can be different. This method is advantageous in many aspects compared with those available. It shows the annual increasing rate clearly which is useful to schedule coming missions. Sustainable development plan should be made based on the increasing rate of specific indexes and corresponding national conditions.

\section{REFERENCES}

[1] What is Sustainable Development? Environmental, economic and social well-being fort oday and tomorrow sustainable development. http://www.iisd.org/sd/.

[2] Kates W. Robert, Thomas M. Parris \& Anthony A. Leiserowitz, 02 Aug 2012. What is Sustainable Development? Goals, Indicators, Values, and Practice. For authors and subscription information. http: //www.tandfonline.com/loi/venv20.

[3] World Commission on Environment and Development. Our Common Future [R].Oxford:Oxford University Press , 1987 .400.

[4] Zhiqiang Zhang, Guodong Cheng, Zhongmin Xu. Sustainable Development Evalu- ation Index, Method and Applicational Research. 2002.

[5] wikipedia:Human Development Index.

[6] Farhad Noorbakhsh. 1998. A Modified Human Development Index. World Devel- opment, Vol.26 (3), pp.517-528.

[7] Shucai Ma, Shu zhang, Yunhong Han. The sustainable development situation of China and comparation with other countries.

[8] The definition of sustainable development.

[9] Rajesh Kumar Singha, H.R. Murtyb, S.K. Guptac, A.K. Dikshitc. An overview of sustainability assessment methodologies.
[10] journal homepage:www.elsevier.com/locate/ecolind.

[11] A healthy city for all: Vancouver's Healthy City Strategy 2014 2025 http://vancouver.ca/people-programs/healthy-city-strategy.aspx.

[12] United Nations Educational, Scientific and Cultural Organization http://www. unesco.org/new/zh/unesco/.

[13] Quantitative evaluation methodology for system coordination development based on distance coordination degree model Ling TANG, Jian ping Ll, Le an YU, Dong hai QIAN,2010.

[14] http://www.doc88.com/p-9774748496891.html.

[15] http://epub.cnki.net/kns/brief/default_result.aspx.

[16] The New Generation of Investment Policies for Sustainable Development:A re- gional training workshop for Southeast Asia Bangkok, Thailand, 27-31 May 2013.

[17] Xiao peng WANG, Yong nian ZENG, Sheng xi DING, Guang chao CAO.Quality Assessment Model for Complex Environment System Based on Refinements to Principal Component Analysis[J]. Systemetic Engineering Theory and Practice,2005,11:114-120.

[18] Xiao peng1 WANG,Guang chao CAO.Model and application of lake and reservoir eutrophication assessment based on multivariate statistical analy- sis[J].Mathematical Statistics and Management,2003,02:30-33.

[19] Yao long DUN, Jun WANG,Yi qiang GUO, Ya nan ZHANG.Sustainability Evalua- tion of Land Consolidation of DaâA ${ }^{-} \mathrm{Z}^{\prime}$ an City Based on AHP-FCE Model[J].China's land science,2014,08:5764.

[20] Rong RONG .Study on the Index System Of Sustainable Development of Coastal Ecotourism[D].Dalian University of Technology ,2008. 